University of Wollongong

Research Online

Faculty of Engineering and Information

Faculty of Engineering and Information

Sciences - Papers: Part A

Sciences

$1-1-2013$

\title{
A secure and effective anonymous user authentication scheme for roaming service in global mobility networks
}

Fentong Wen

University of Wollongong, ftong@uow.edu.au

Willy Susilo

University of Wollongong, wsusilo@uow.edu.au

Guomin Yang

University of Wollongong, gyang@uow.edu.au

Follow this and additional works at: https://ro.uow.edu.au/eispapers

Part of the Engineering Commons, and the Science and Technology Studies Commons

Research Online is the open access institutional repository for the University of Wollongong. For further information contact the UOW Library: research-pubs@uow.edu.au 


\title{
A secure and effective anonymous user authentication scheme for roaming service in global mobility networks
}

\begin{abstract}
In global mobility networks, anonymous user authentication is an essential task for enabling roaming service. In a recent paper, Jiang et al. proposed a smart card based anonymous user authentication scheme for roaming service in global mobility networks. This scheme can protect user privacy and is believed to have many abilities to resist a range of network attacks, even if the secret information stored in the smart card is compromised. In this paper, we analyze the security of Jiang et al.'s scheme, and show that the scheme is in fact insecure against the stolen-verifier attack and replay attack. Then, we also propose a new smart card based anonymous user authentication scheme for roaming service. Compared with the existing schemes, our protocol uses a different user authentication mechanism, which does not require the home agent to share a static secret key with the foreign agent, and hence, it is more practical and realistic. We show that our proposed scheme can provide stronger security than previous protocols.
\end{abstract}

\section{Keywords}

era2015, roaming, service, scheme, authentication, user, networks, anonymous, mobility, effective, secure, global

\section{Disciplines}

Engineering | Science and Technology Studies

\section{Publication Details}

Wen, F., Susilo, W. \& Yang, G. (2013). A secure and effective anonymous user authentication scheme for roaming service in global mobility networks. Wireless Personal Communications: an international journal, 73 (3), 993-1004. 


\title{
A Secure and Effective Anonymous User Authentication Scheme for Roaming Service in Global Mobility Networks
}

\author{
Fengtong Wen · Willy Susilo · Guomin \\ Yang
}

Received: date / Accepted: date

\begin{abstract}
In global mobility networks, anonymous user authentication is an essential task for enabling roaming service. In a recent paper, Jiang et al. proposed a smart card based anonymous user authentication scheme for roaming service in global mobility networks. This scheme can protect user privacy and is believed to have many abilities to resist a range of network attacks, even if the secret information stored in the smart card is compromised. In this paper, we analyze the security of Jiang et al.'s scheme, and show that the scheme is in fact insecure against the stolen-verifier attack and replay attack. Then, we also propose a new smart card based anonymous user authentication scheme for roaming service. Compared with the existing schemes, our protocol uses a different user authentication mechanism, which does not require the home agent to share a static secret key with the foreign agent, and hence, it is more practical and realistic. We show that our proposed scheme can provide stronger security than previous protocols.
\end{abstract}

Keywords Roaming $\cdot$ Authentication · Cryptanalysis $\cdot$ Security $\cdot$ Smart card

F.Wen

1.School of Mathematical Sciences, University of Jinan, 250022, China

2.School of Computer Science and Software Engineering, University of Wollongong, NSW 2522, Australia.

Tel.: +86-15666966618

E-mail:wftwq@163.com

present address: University of Wollongong, NSW 2522, Australia.

W.Susilo

School of Computer Science and Software Engineering, University of Wollongong, NSW 2522, Australia.

G.Yang

School of Computer Science and Software Engineering, University of Wollongong, NSW 2522, Australia. 


\section{Introduction}

Nowadays, with the fast development of mobile technologies, Global Mobility Networks (GLOMONETs) have become widely available and interconnected. To provide global roaming service for a mobile user, remote authentication is an essential requirement. A typical remote authentication scenario involves three parties, namely a Mobile User (MU), a Foreign Agent(FA) and a Home Agent(HA). When a mobile user MU roams into a foreign network, the foreign agent FA authenticates the roaming user with the help of the user's home agent $\mathrm{HA}[1,10,11]$.

During the roaming process in GLOMONET, the mobile user MU is very much concerned about its privacy protection. The user's identity should be protected and his/her location and activities should be kept unlinkable. It is desirable to keep mobile users' identities anonymous in the remote user authentication process. In recent years, many anonymous authentication scheme (e.g. $[22,12,21,2,13,6,14,19,7,8])$ have been proposed for roaming services in GLOMONET. However, most of the existing protocols were broken shortly after they were proposed.

Mutual Authentication is a very important security feature. It requires that the client and server prove their respective identities to each other before performing any application functions. Recently, many research work(e.g.[22, $4,8,23,24,20])$ have been done in the design and analysis of mutual authentication protocols. However, some of them have been proved to be insecure against known attacks.

In 2012, Jiang et al. [9] pointed out the security flaws in some previous smart card based anonymous user authentication protocols. In order to remedy those weaknesses, Jiang et al. proposed an authentication scheme based on quadratic residue assumption, which is very efficient. Hence, Jiang et al.'s scheme seems to be a very good candidate authentication protocol for adoption in practice. Therefore, it is very interesting to analyze this scheme in detail to ensure that the security claims that are provided by the authors hold. It is unfortunate that we found some serious flaws in their scheme. In particular, the scheme is insecure due to two important issues namely the stolen-verifier attack [17] and it cannot resist against replay attacks.

Our Contributions. The contributions of this paper are twofold. First, we show there exist several serious security flaws in Jiang et al.'s anonymous user authentication scheme, which are described as follows: 1) It is vulnerable to stolen-verifier attack. An attacker who has stolen the verifier table can obtain the session key $S K$ and can impersonate the FA to fool the MU. 2) It also cannot resist replay attacks. Although the attacker cannot get the MU's session key shared with the FA, he/she can impersonate the MU to login the FA. 3) It is vulnerable to denial of service attack.

Second, we propose a new smart card based anonymous user authentication protocol for roaming service in global mobility networks. Our protocol makes 
use of a user authentication mechanism which is different from the previous approaches and can successfully prevent different kinds of network attacks.

Organization of the Paper. The rest of this paper is organized as follows. In the next section, we provide some mathematical preliminaries, which will be used throughout the paper. In Section 3, we briefly review Jiang et al.'s scheme. Subsequently, we show its weaknesses in Section 4. Then, we proceed with proposing our scheme in Section 5, together with analyzing its security in Section 6. In Section 7, we compare the performance of our new protocol with the previous schemes. Section 8 concludes the paper.

\section{Mathematical Preliminaries}

In this section, we discuss three computational problem: Quadratic residue problem, discrete logarithm problem and computational Diffie-Hellman problem, which will be used throughout the paper.

\subsection{Quadratic Residue Problem}

Assume that $n=p q$, where $p$ and $q$ are two large primes. If $y=x^{2} \bmod n$ has a solution, i.e., there exists a square root for $y$, then $y$ is called a quadratic residue $\bmod n$. The set of all quadratic residue numbers in $[1, n-1]$ is denoted by $Q R_{n}$. Then the quadratic residue problem states that, for $y \in Q R_{n}$, it is hard to find $x$ without the knowledge of $p$ and $q$ due to the difficulty of factoring $n[16]$.

\subsection{Discrete Logarithm Problem}

Let $G$ be a finite group, $g \in G$ with order $n, y \in G_{g}$. The problem is to find the smallest integer $x \in\{1, \ldots, n-1\}$ such that $g^{x}=y(\bmod n)$. It is easy to compute the discrete exponentiation $y=g^{x}(\bmod n)$ given $g, x, n$, but it is computationally infeasible to determine $x$ given $y, g, n$, when $n$ is large.

\subsection{Computational Diffie-Hellman Problem}

Consider a cyclic group $G$ with order $q$. The $\mathrm{CDH}$ problem states that, given $g, g^{x}, g^{y}$ for a generator $g$ and random number $x, y \in\{1,2, \ldots, q-1\}$, it is computationally intractable to compute the value $g^{x y}$.

The notations that will be used throughout this paper are listed in Table 1. 
Table 1 Notations

\begin{tabular}{cl}
\hline$M U$ & The Mobile user \\
$I D_{M U}$ & Identity of MU \\
$P W_{M U}$ & Password of MU \\
$H A$ & Home agent of MU \\
$I D_{H A}$ & Identity of HA \\
$F A$ & Foreign agent of MU roamed \\
$I D_{F A}$ & Identity of FA \\
$x$ & master secret key stored in HA \\
$c t r_{M U}$ & A counter of MU \\
$S K$ & Session key shared between A and B \\
$h(\cdot)$ & A secure collision-free one-way hash function \\
$A \oplus B$ & XOR operation of A and B \\
$A \| B$ & Data A concatenates with data B \\
\hline
\end{tabular}

\section{Review of Jiang et al.'s scheme}

Jiang et al.'s smart card based anonymous authentication scheme comprises three phases, namely the registration phase, the login and authentication phase and the password update phase. Each foreign agent shares a unique long-term $K_{F H}$ with HA.

\subsection{Registration Phase}

Step 1. The MU sends his/her $I D_{M U}$ to $\mathrm{HA}$ via a secure communication channel. HA computes $K_{M U}=h\left(I D_{M U} \| x\right)$ using the secret random number $x$.

Step 2. HA personalizes the smart card with $h(\cdot), K_{M U}, n$ and sends it to MU, where $n$ is the modulus in the Quadratic Residue Problem.

Step 3. After receiving the smart card, the MU computes $K_{M U}^{*}=K_{M U} \oplus$ $h\left(I D_{M U} \| P W_{M U}\right)$ and replaces $K_{M U}$ with $K_{M U}^{*}$. Finally, the smart card contains $h(\cdot), K_{M U}^{*}, n$.

\subsection{Login and Authentication Phase}

Step 1. MU inserts his/her smart card into the device and enters his/her identity $I D_{M U}$ and password $P W_{M U}$. The smart card computes $K_{M U}=$ $K_{M U}^{*} \oplus h\left(I D_{M U} \| P W_{M U}\right)$. Then the smart card generates a random number $n_{M U}$ and computes $V_{1}=\left(I D_{M U}\left\|K_{M U}\right\| n_{M U} \| I D_{F A}\right)^{2} \bmod n$. Finally, MU sends a login message $M_{1}=\left(V_{1}, I D_{H A}\right)$ to FA.

Step 2. Upon receiving $M_{1}$, FA generates a random number $n_{F A}$ and computes $V_{2}=h\left(M_{1}\left\|n_{F A}\right\| K_{F H}\right)$. Subsequently, FA sends the message $M_{2}=$ $\left(M_{1}, I D_{F A}, n_{F A}, V_{2}\right)$ to HA. 
Step 3. After receiving $M_{2}$, HA checks whether $I D_{F A}$ is the identity of an ally. If it is true, HA compares $V_{2}$ with $V_{2}^{*}=h\left(M_{1}\left\|n_{F A}\right\| K_{F H}\right)$. If $V_{2} \neq V_{2}^{*}$, HA terminates the protocol. Otherwise, HA solves $V_{1}$ by using the Chinese Remainder Theorem with $p$ and $q$ to get $I D_{M U}, K_{M U}, n_{M U}, I D_{F A}$. Then, HA computes $h\left(I D_{M U} \| x\right)$ and compares it with the received $K_{M U}$. If they are equal, the authenticity of MU is ensured. Also, HA believes FA is the target foreign agent of MU. After that, HA computes $S K=h\left(h\left(I D_{M U} \| x\right)\left\|I D_{F A}\right\| n_{M U}\right.$ $\left.\| n_{F A}\right), V_{3}=S K \oplus h\left(K_{F H} \| n_{F A}\right)$ and $V_{4}=h\left(V_{3} \| K_{F H}\right)$, where $S K$ is the session key between FA and MU. Finally, HA sends $M_{3}=\left(V_{3}, V_{4}\right)$ to FA.

Step 4. After receiving the message $M_{3}$, FA computes $V_{4}^{*}=h\left(V_{3} \| K_{F H}\right)$ and checks whether $V_{4}^{*}=V_{4}$. If it is valid, FA computes $S K=V_{3} \oplus h\left(K_{F H} \| n_{F A}\right)$ and $V_{5}=h\left(S K \| n_{F A}\right)$, and sends $M_{4}=\left(n_{F A}, V_{5}\right)$ to MU.

Step 5. Upon receiving $M_{4}$, MU computes $S K^{*}=h\left(h\left(I D_{M U} \| x\right)\left\|I D_{F A}\right\| n_{M U}\right.$ $\left.\| n_{F A}\right)$. Then MU computes $V_{5}^{*}=h\left(S K^{*} \| n_{F A}\right)$ and checks whether it is equal to $V_{5}$. If it is true, then MU establishes trust with FA; otherwise, the authentication fails.

\subsection{Password Change Phase}

When MU wants to renew a password, MU inserts his/her smart card into the terminal and enters his/her identity $I D_{M U}^{*}$, the old password $P W_{M U}$, and the new password $P W_{M U}^{* *}$. Then the smart card retrieves $K_{M U}=K_{M U}^{*} \oplus$ $h\left(I D_{M U}^{*} \| P W_{M U}\right)$ and computes $K_{M U}^{* *}=K_{M U} \oplus h\left(I D_{M U}^{*} \| P W_{M U}^{* *}\right)$. Finally, the smart card stores $K_{M U}^{* *}$ in place of $K_{M U}^{*}$

\section{Security Analysis of Jiang et al.'s Scheme}

\subsection{Security against Stolen-Verifier Attacks}

In Jiang et al.'s scheme, it is assumed that each foreign agent shares a unique long-term key $K_{F H}$ with HA which should be stored in the verifier table of HA's database. In case the verifier table in the HA's database is leaked out or stolen by an attacker, the attacker can compute $S K$ corresponding to any user $\mathrm{MU}$ and can also proceed to spoofing attack. This is illustrated as follows.

\subsubsection{Computing SK}

Step 1. Eavesdrops a login request message $M_{2}=\left(M_{1}, I D_{F A}, n_{F A}, V_{2}\right)$ and the corresponding message $M_{3}=\left(V_{3}, V_{4}\right)$.

Step 2. Computes $h\left(K_{F H} \| n_{F A}\right)$ using the stolen long-term key $K_{F H}$ and $n_{F A}$. Step 3. Computes $S K=V_{3} \oplus h\left(K_{F H} \| n_{F A}\right)$.

It can be seen that Jiang et al.'s protocol does not achieve perfect forward secrecy if the attacker obtains the FA's long-term key $K_{F H}$ shared with HA. 


\subsubsection{Spoofing Attack}

Step 1. Intercepts a login request message $M_{2}=\left(M_{1}, I D_{F A}, n_{F A}, V_{2}\right)$ and chooses a random number $n_{F A}^{\prime}$, and then computes $V_{2}^{\prime}=h\left(M_{1}\left\|n_{F A}^{\prime}\right\| K_{F H}\right)$ using the stolen long-term key $K_{F H}$ and $n_{F A}^{\prime}$. Sends $M_{2}^{\prime}=\left(M_{1}, I D_{F A}, n_{F A}^{\prime}, V_{2}^{\prime}\right)$ to the HA.

Step 2. The HA authenticates the MU and FA following the step 3 of Jiang et al.'s scheme and sends message $M_{3}^{\prime}=\left(V_{3}^{\prime}, V_{4}^{\prime}\right)$ to the attacker, where $V_{3}^{\prime}=$ $S K^{\prime} \oplus h\left(K_{F H} \| n_{F A}^{\prime}\right), V_{4}^{\prime}=h\left(V_{3}^{\prime} \| K_{F H}\right), S K^{\prime}=h\left(h\left(I D_{M U} \| x\right)\left\|I D_{F A}\right\| n_{M U}\right.$ $\left.\| n_{F A}^{\prime}\right)$.

Step 3. After receiving the message $M_{3}^{\prime}=\left(V_{3}^{\prime}, V_{4}^{\prime}\right)$, the attacker computes $S K^{\prime}=V_{3}^{\prime} \oplus h\left(K_{F H} \| n_{F A}^{\prime}\right), V_{5}^{\prime}=h\left(S K^{\prime} \| n_{F A}^{\prime}\right)$ and sends $M_{4}^{\prime}=\left(n_{F A}^{\prime}, V_{5}^{\prime}\right)$ to the MU.

Step 4. The MU computes $S K^{*}=h\left(h\left(I D_{M U} \| x\right)\left\|I D_{F A}\right\| n_{M U} \| n_{F A}^{\prime}\right), V_{5}^{*}=$ $h\left(S K^{*} \| n_{F A}^{\prime}\right)$ and conforms $V_{5}^{*}=V_{5}^{\prime}$. The MU believes that the attacker is the FA.

Hence, it is clear that the attacker can impersonate the FA whenever he/she likes.

\subsection{Security against Replay Attacks}

In Jiang et al.'s scheme, during the login and authentication phase, the MU sends the login message $M_{1}=\left(V_{1}, I D_{H A}\right)$ to FA via a public channel, where $V_{1}=\left(I D_{M U}\left\|K_{M U}\right\| n_{M U} \| I D_{F A}\right)^{2} \bmod n$. Suppose an attacker intercepts this message and sends another message $M_{1}^{\prime}=M_{1}$ in the next time to FA. Then after receiving this message during login and authentication phase, the FA generates a random number $n_{F A}^{\prime}$ and computes $V_{2}^{\prime}=h\left(M_{1}^{\prime}\left\|n_{F A}^{\prime}\right\| K_{F H}\right)$. Subsequently, FA sends the message $M_{2}^{\prime}=\left(M_{1}^{\prime}, I D_{F A}, n_{F A}^{\prime}, V_{2}^{\prime}\right)$ to HA. After receiving $M_{2}^{\prime}$, then HA also processes this message and ensures that the attacker is a legal user. Although the attacker cannot obtain the session key $S K$, he/she can impersonate the MU to login to the FA. As a result, Jiang et al.'s scheme fails to provide protection against a strong replay attack.

\subsection{Security against Denial of Service Attack}

In the password change phase, there is no verification of old password before the new password is updated. If an attacker manages to gain temporary access to MU's smart card, he/she can launch a denial of service attack as follows:

1) Inserts MU's smart card into a card reader and initiates a password change request.

2) Submits two random strings $R_{1}, R_{2}$ as MU's old password and new password. 
3) The smart card computes $K_{M U}^{* *}=h\left(I D_{M U} \| x\right) \oplus h\left(I D_{M U} \| P W_{M U}\right) \oplus$ $h\left(I D_{M U} \| R_{1}\right) \oplus h\left(I D_{M U} \| R_{2}\right)$ and updates $K_{M U}^{*}$ with $K_{M U}^{* *}$.

Once the value of $K_{M U}^{*}$ is updated, the MU cannot login successfully even if he/she gets his/her smart card back because $K_{M U}^{* *} \oplus h\left(I D_{M U} \| P W_{M U}\right) \neq$ $h\left(I D_{M U} \| x\right)$, and since then MU's login request will be denied by HA during the login and authentication phase.

\section{The Proposed Scheme}

In this section, we propose a new authentication scheme with privacy preservation for global mobility networks. Notably, the new scheme makes use of a counter-based authentication mechanism to resist against replay attack. In order to resist against stolen-verifier attack [17], we replace the static long-term key $K_{F H}$ with a Diffie-Hellman key. The new scheme can also resist against a range of attacks such as offline password guessing attacks and denial of service attack, even if the smart card is stolen.

Before the system begins, HA generates two secret large primes $p, q$ and computes the number $n=p q$. The home agent HA chooses a multiplication group $G$ and an element $g \in G$ with order $q^{\prime}$, where $p^{\prime}$ and $q^{\prime}$ are public large primes and $p^{\prime}=2 q^{\prime}+1$ is the modulus for the group $G\left(p^{\prime}, q^{\prime}\right.$ is different from $p, q)$. The HA selects a private key $S_{H A}=a\left(<q^{\prime}\right)$ and computes the public key $P_{H A}=g^{a} \bmod p^{\prime}$. Similarly, the foreign agent FA selects a private key $S_{F A}=b\left(<q^{\prime}\right)$ and computes the public key $P_{F A}=g^{b} \bmod p^{\prime}$. The new protocol has three phases: registration, login and authentication, and password change.

\subsection{Registration Phase}

Step 1. The MU chooses his/her own identity $I D_{M U}$ and password $P W_{M U}$, and generates a large random number $d$. He/she then computes the hash value $f=h\left(I D_{M U}\left\|P W_{M U}\right\| d\right)$, and sends the registration message $I D_{M U}, f$ to the HA via a secure channel.

Step 2. HA computes $K_{M U}^{*}=h\left(I D_{M U} \| x\right) \oplus f$ using the secret random number $x$. HA then initiates a counter $c t_{M U}=0$ for $\mathrm{MU}$ and creates a record $\left(I D_{M U}, c t r_{M U}\right)$ in its database. HA then personalizes the smart card with $h(\cdot), K_{M U}^{*}, c t r_{M U}, n$ and sends it to MU.

Step 3. After receiving the smart card, the MU computes $f^{*}=h\left(I D_{M U} \oplus\right.$ $\left.P W_{M U} \oplus d\right)$ and stores $f^{*}$ in the smart card. Finally, the smart card contains $h(\cdot), K_{M U}^{*}, f^{*}, n, d, c \operatorname{ct}_{M U}$. 
5.2 Login and Authentication Phase

Step 1. $M U \rightarrow F A: M_{1}$. MU inserts his/her smart card into the device and enters his/her identity $I D_{M U}$ and password $P W_{M U}^{\prime}$. The smart card computes $f^{\prime}=h\left(I D_{M U} \oplus P W_{M U}^{\prime} \oplus d\right)$ and verifies whether $f^{*}=f^{\prime}$ or not. If $f^{*} \neq f^{\prime}$, the login phase terminates immediately. Otherwise, the smart card computes $K_{M U}=K_{M U}^{*} \oplus h\left(I D_{M U}\left\|P W_{M U}\right\| d\right)$. Then MU generates a random number $n_{M U}$ and computes $c t r_{M U}=c t r_{M U}+1, V_{1}=\left(I D_{M U}\left\|K_{M U}\right\| n_{M U} \| c t r_{M U}\right.$ $\left.\| I D_{F A}\right)^{2} \bmod n$. Finally, MU sends a login message $M_{1}=\left(V_{1}, c t r_{M U}, I D_{H A}\right)$ to FA.

Step 2. FA $\rightarrow H A: M_{2}$. FA computes $K=P_{H A}^{b} \bmod p^{\prime}=g^{a b} \bmod p^{\prime}, V_{2}=$ $h\left(V_{1}\left\|c t r_{M U}\right\| K\right)$. Subsequently, FA sends the message $M_{2}=\left(M_{1}, I D_{F A}, V_{2}\right)$ to HA.

Step 3. $H A \rightarrow F A: M_{3}$. HA checks whether $I D_{F A}$ is the identity of an ally. If it is true, HA compares $K=P_{F A}^{a} \bmod p^{\prime}=g^{a b} \bmod p^{\prime}$ and $V_{2}^{*}=$ $h\left(V_{1}\left\|c t r_{M U}\right\| K\right)$. If $V_{2} \neq V_{2}^{*}$, HA terminates the protocol. Otherwise, HA solves $V_{1}$ by using the Chinese Remainder Theorem with $p$ and $q$ to get $I D_{M U}, K_{M U}, n_{M U}, c \operatorname{tr}_{M U}, I D_{F A}$. Then, the HA verifies the retrieved $c r_{M U}$ with the stored $c t r_{M U}^{\prime}$ corresponding to $I D_{M U}$. If $c t r_{M U}>c t r_{M U}^{\prime}$, then the HA proceeds to the next step. Otherwise, the HA rejects this message and considers it as a replayed message. After that, HA computes $h\left(I D_{M U} \| x\right)$ and compares it with the received $K_{M U}$. If they are equal, the authenticity of MU is ensured, and the HA replaces $c t r_{M U}^{\prime}$ with the new counter $c t r_{M U}$ in its database. Also, HA believes FA is the target foreign agent of MU. HA computes $S K=h\left(h\left(I D_{M U} \| x\right)\left\|I D_{F A}\right\| n_{M U} \| c t r_{M U}\right), V_{3}=S K \oplus h(K)$ and $V_{4}=h\left(V_{3} \| K\right)$, where $S K$ is the session key between FA and MU. Finally, HA sends $M_{3}=\left(V_{3}, V_{4}\right)$ to FA.

Step 4. FA $\rightarrow M U: M_{4}$. FA computes $V_{4}^{*}=h\left(V_{3} \| K\right)$ and checks whether $V_{4}^{*}=V_{4}$. If it is valid, FA computes $S K=V_{3} \oplus h(K)$ and $V_{5}=h\left(S K \| P_{F A}\right)$, and sends $M_{4}=V_{5}$ to $\mathrm{MU}$.

Step 5. Upon receiving $M_{4}$, MU computes $S K^{*}=h\left(h\left(I D_{M U} \| x\right)\left\|I D_{F A}\right\| n_{M U}\right.$ $\left.\| c t r_{M U}\right)$. Then MU computes $V_{5}^{*}=h\left(S K^{*} \| P_{F A}\right)$ and checks whether $V_{5}^{*}=$ $V_{5}$. If they are equal, then MU establishes trust with FA; otherwise, the authentication fails.

\subsection{Password Change Phase}

When MU wants to renew a password, MU inserts his/her smart card into the terminal and enters his/her identity $I D_{M U}$, the old password $P W_{M U}^{\text {old }}$, and the new password $P W_{M U}^{n e w}$. The smart card computes $f^{\prime}=h\left(I D_{M U} \oplus P W_{M U}^{\text {old }} \oplus d\right)$, and then verifies whether $f^{*}=f^{\prime}$ or not. If $f^{*} \neq f^{\prime}$, the password change phase is terminated immediately. Otherwise, the smart card retrieves $K_{M U}=K_{M U}^{*} \oplus$ 


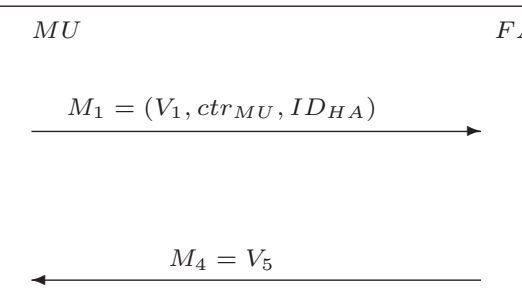

Fig. 1 Message Flows in login and Authentication Phase.

$h\left(I D_{M U}\left\|P W_{M U}^{o l d}\right\| d\right)$ and computes $K_{M U}^{* *}=K_{M U} \oplus h\left(I D_{M U}\left\|P W_{M U}^{n e w}\right\| d\right)$. Finally, the smart card stores $K_{M U}^{* *}$ in place of $K_{M U}^{*}$.

\section{Security Analysis of The Proposed Scheme}

In this section, we analyze the security of the proposed scheme and show that it can resist different types of attacks and also provides user anonymity.

\subsection{User Anonymity}

It is very important and necessary for a secure authentication protocol to provide user anonymity $[3,5]$. From Figure 1, we can see that the communication transcript reveals no information about the identity $I D_{M U}$ of the user. In fact, $I D_{M U}$ is concealed in $V_{1}$, only the person who knows the value of $p, q$ can solve the quadratic residue problem to obtain $I D_{M U}$. In our scheme, the secret value $p, q$ is stored by HA. Therefore, the attacker including the FA cannot identify the MU from the login message.

\subsection{Security against Replay Attacks [18]}

In our scheme, we used the counter based authentication mechanism to prevent replay attacks. If the adversary replays the previous login message, then HA will detect the attack when examining the counter ctr $_{M U}$ of the user MU. The concrete step is as follows: During the login and authentication phase, when the HA receives a message $M_{2}^{\prime}=\left(M_{1}^{\prime}, I D_{F A}, V_{2}^{\prime}\right)$, it verifies the retrieved counter ctr $_{M U}$ with the stored counter $c r_{M U}^{\prime}$ corresponding to the $I D_{M U}$. If the message $M_{2}^{\prime}=\left(M_{1}^{\prime}, I D_{F A}, V_{2}^{\prime}\right)$ is a replayed message, then the HA will find that $c t r_{M U}^{\prime} \geq c r_{M U}$. The HA will simply reject this message. Hence, our scheme prevents the replay attacks through the use of counter.

\subsection{Security against Impersonation Attacks [15]}

We consider three types of impersonation attacks, namely MU impersonation attack, FA impersonation attack, and HA impersonation attack. 
MUImpersonation. In order to impersonate the $\mathrm{MU}$, the adversary must obtain the value of $I D_{M U}, K_{M U}$. We then consider two situations: (1) the adversary compromises the user identity and password but the smart card is secure; and (2) the adversary compromises the smart card but the user identity and password are secure.

In the first case, it is obvious that adversary is unable to obtain the value of $K_{M U}$ since the smart card is secure. Notice that $K_{M U}$ is concealed in $K_{M U}^{*}=K_{M U} \oplus h\left(I D_{M U}\left\|P W_{M U}\right\| d\right)$ in the smart card. Since the smart card is secure, the adversary is unable to obtain $\left(K_{M U}^{*}, d\right)$ or $K_{M U}$.

In the second case, when the smart card is stolen and compromised, the adversary can learn the values of $\left(h(\cdot), K_{M U}^{*}, f^{*}, n, d, c t r_{M U}\right)$ in the smart card. However, this time the adversary knows neither $I D_{M U}$ nor $P W_{M U}$, and again he/she cannot computes the value of $K_{M U}$. We must also consider offline password guessing attacks in this case, that is the adversary uses a brute force search to find out the correct password. Let the adversary select randomly an identity candidate $I D_{M U}^{\prime}$ and a password candidate $P W_{M U}^{\prime}$. Then the adversary can compute $f^{\prime}=h\left(I D_{M U}^{\prime} \oplus P W_{M U}^{\prime} \oplus d\right)$ and then compare $f^{\prime}$ with $f^{*}$. If they are equal, the adversary may think that $P W_{M U}^{\prime}$ and $I D_{M U}^{\prime}$ as the correct password and real identity of the MU. In fact, the message pair $\left(I D_{M U}^{\prime}, P W_{M U}^{\prime}\right)$ which satisfy the above equality is not unique. Even if this attack can be carried out in the offline manner by repeatedly trying the next identity candidate and password candidate, it is still a difficult problem for the adversary to obtain the real $I D_{M U}, P W_{M U}$ of the MU.

$F A / H A$ Impersonation. Since FA and HA use Diffie-Hellman keys to authenticate each other, the adversary is unable to impersonate either of them due to the intractability of the Diffie-Hellman problem. Also, the attacker cannot impersonates FA to fool MU. Since he/she does not posses the secret $x$ and $I D_{M U}, n_{M U}$, the attacker cannot compute $V_{5}=h\left(S K \| P_{F A}\right)=$ $h\left(h\left(h\left(I D_{M U} \| x\right)\left\|I D_{F A}\right\| n_{M U} \| c t r_{M U}\right) \| P_{F A}\right)$. On the other hand, it is impossible for him/her to obtain the session key $S K$ by computing $S K=V_{3} \oplus h(K)$ due to the difficulty of solving Diffie-Hellman problem.

\subsection{Secure Key Establishment [15]}

At the end of the protocol, the MU, FA and HA will establish a common session key $S K=h\left(h\left(I D_{M U} \| x\right)\left\|I D_{F A}\right\| n_{M U} \| c t r_{M U}\right)$. Since the attacker does not know the value of $x$, he/she cannot compute the $S K$ directly. Even if the previous session keys are disclosed, the attacker cannot obtain any future session key due to the security of one-way hash function.

\subsection{Protection against Attacks in Section 4}

In this section, we show how our new scheme overcomes the security weaknesses presented in Section 4. 
1) In our proposed scheme, there is no verifier table in HA's database to store the secret information of MU or FA. Thus, the attacker cannot launch stolen-verifier attack [17].

2) In order to prevent replay attack [18], we used the counter based authentication mechanism. HA can detect the attack by examining the counter ctr $_{M U}$ of the user MU.

3) In order to prevent denial of service attack [25], the smart card will verify whether the $P W_{M U}$ entered by $\mathrm{MU}$ is correct or not in the login phase and password update phase.

\section{Performance Comparison}

We compare our new scheme with two recently proposed smart card based anonymous user authentication schemes due to He et al. [8] and Jiang et al. [9]. In Table 2, we provide the comparison based on the key security of these schemes, while we compare their efficiency in terms of computation and communication cost in Table 3. The following notation are used in Table 3. $t_{h}$ : The time complexity of the hash computation; $t_{m}$ : The time complexity of the modular squaring computation; $t_{q r}$ : The time complexity of computing a square root modulo $n ; t_{m e}$ : The time complexity of computing modular exponentiation; $t_{\text {sym }}$ : The time complexity of symmetric encryption/decryption; $t_{\text {asym }}$ : The time complexity of encryption/decryption or signature using asymmetric cryptosystem.

From Table 2, we can conclude that our proposed scheme provides better security and usability than the other two schemes. He et al.'s scheme [8] does not satisfy any of the criteria listed in table 2. Jiang et.al.'s scheme [9] does not satisfy four of the nine criteria. Our scheme can achieve all the criteria listed in table 2. In particular, one special feature of our scheme is that we do not require the HA to store some secret information that is shared with FA in his/her database, which enhances our scheme's security strength to resist against different attacks.

In Table 3, we summarize the efficiency comparison between our scheme and other schemes in $[9,8]$. From the table 3 , it is easy to see that our scheme is more efficient than He et al.'s scheme [8]. Our scheme requires two extra Modular Exponentiation for FA and HA as compared with Jiang et al.'s scheme. It is not a problem because the FA and the HA are powerful servers. On the other hand, our scheme achieves stronger security than the previous solutions, as is shown in Table 2 .

\section{Conclusion}

In this paper, we discussed several security weaknesses in a recently proposed smart card based privacy preservation user authentication scheme by Jiang et al. We showed that this scheme is vulnerable to stolen-verifier attacks, replay 
Table 2 Security and Usability Comparison

\begin{tabular}{cccc}
\hline Feature & He et al. [8] & Jiang [9] & Ours \\
\hline Correct Password Update & No & No & Yes \\
Stolen-verifier-table resistance & No & No & Yes \\
User Anonymity & No & Yes & Yes \\
Mutual Authentication & No & Yes & Yes \\
Strong Replay Resistance & No & No & Yes \\
Key Agreement & No & Yes & Yes \\
Password protection & No & Yes & Yes \\
Two-factor security & No & Yes & Yes \\
Denial of service resistance & No & No & Yes \\
\hline
\end{tabular}

Table 3 Efficiency Comparison

\begin{tabular}{cccc}
\hline & Jiang et al. [9] & He et al. [8] & Ours \\
\hline C1 & $3 t_{h}+t_{m}$ & $10 t_{h}+2 t_{\text {sym }}$ & $3 t_{h}+t_{m}$ \\
C2 & $4 t_{h}$ & $t_{h}+t_{\text {asym }}$ & $3 t_{h}+t_{m e}$ \\
C3 & $5 t_{h}+t_{q r}$ & $4 t_{h}+2 t_{\text {sym }}+4 t_{\text {asym }}$ & $5 t_{h}+t_{m e}+t_{q r}$ \\
C4 & 1 & 1 & 1 \\
C5 & 1 & 1 & 1 \\
C6 & 4 & 5 & 4 \\
C7 & 6 & 6 & 5 \\
\hline
\end{tabular}

C1: Computation cost of the MU

C2: Computation cost of the FA

C3: Computation cost of the HA

C4: Communication rounds between the MU and FA

C5: Communication rounds between the FA and HA

C6: Total messages transmitted between the MU and FA

C7: Total messages transmitted between the FA and HA

attacks, and denial of service attack. In order to withstand its security flaws, subsequntly we proposed a new smart card based user authentication scheme with user's anonymity for roaming service which can achieve stronger security. Our scheme does not require HA to store a verifier-table in its database and uses counter based authentication mechanism to prevent replay attacks.

\section{References}

1. Chang, C., Lee, J. \& Chang, Y. (2005). Efficient authentication protocols of GSM. Computer Communications, 28(8), 921-928.

2. Chang, C., Lee, C. \& Chiu, Y. (2009). Enhanced authentication scheme with anonymity for roaming service in global mobility networks, Computer Communications, 32(4), 611618.

3. Chaum, D. (1985). Security Withont Identification: Trunsactions Systems to Make Big Brother Obsolete. Commun. ACM 28(10), 1030-1044.

4. Das A. 2012. A secure and effective user authentication and privacy preserving protocol with smart cards for wireless communications. Networking Science, doi:10.1007/S13119012-0009-8. 
5. ETSI TS 102 165-1 V4.1.1 Telecommunications and Internet Protocol Harmonization Over Networks (TIPHON) Release 4; Protocol Framework Definition; Methods and Protocols for Security; Part 1: Threat Analysis.

6. Fatemi, M., Salimi, S., \& Salahi, A. (2010). Anonymous roaming in universal mobile telecommunication system mobile networks. IET Information Security, 4(2), 93-103.

7. He, D., Ma, M., Zhang, Y., \& Chen, C. (2010). A strong user authentication scheme with smart cards for wireless communications. Computer Communications, 34(3), 367-374.

8. He, D., Chan, S., Chen, C., \& Bu, J. (2011). Design and validation of an efficient authentication scheme with anonymity for roaming service in global mobility networks. Wireless Personal Communications, 61,465-476.

9. Jiang, Q., Ma, J., Li, G., \& Yang, L. (2012). An Enhanced Authentication Scheme with Privacy Preservation for Roaming Service in Global Mobility Networks. Wireless Personal Communications. doi:10.1007/s11277-012-0535-4.

10. Lee, C. Hwang, M., \& Liao, I. (2008). A new authentication protocol based on pointer forwarding for mobile communications. Wireless Communication and Mobile Computing, 8(5), 661-672.

11. Lee, C., Hwang, M., \& Yang, W. (2003). Extension of authentication protocol for GSM. IEE Proceedings Communications, 150(2), 91-95.

12. Lee, C., Hwang, M., \& Liao, I. (2006). Security enhancement on a new authentication scheme with anonymity for wireless environments. IEEE Transactions on Industrial Electronics, 53(5), 1683-1686.

13. Lee, T., Chang, C., \& Hwang, T. (2005). Private authentication techniques for the global mobility network. Wireless Personal Communications, 35(4), 329-336.

14. Lee, J., Chang, J., \& Lee, D. (2009). Security flaw of authentication scheme with anonymity for wireless communications. IEEE Communications Letters, 13(5), 292-293.

15. Menezes A., Van Oorschot P., Vanstone S. (1997). Handbook of applied cryptography. CRC, Boca Raton, FL.

16. Rosen, K. (1988). Elementary number theory and its applications. Reading, MA: Addison-Wesley.

17. Liao I., Lee C. \& Hwang M. (2006). A password authentication scheme over insecure networks, J. Comput. System Sci., 72(4), 727-740.

18. Syverson P. (1994). A taxonomy of replay attacks. In Proc. IEEE Computer Security Foundations Workshop VII, pp.131-136.

19. Wang, R., Juang, W., \& Lei, C. (2009). A robust authentication scheme with user anonymity for wireless environments. International Journal of Innovative Computing, Information and Control, 5(4), 1069-1080. http://grouper.ieee.org/groups/1363/

20. Wang, R., Juang, W., \& Lei, C. (2010). Robust authentication and key agreement scheme preserving the privacy of secret key. Computer Communications. doi:10.1016/j.comcom.2010.04.005.

21. Wu, C., Lee, B., \& Tsaur, W. (2008). A secure authentication scheme with anonymity for wireless communications, IEEE Communications Letters, 12(10), 722-723.

22. Zhu, J. \& Ma, J. (2004). A new authentication scheme with anonymity for wireless environments. IEEE Transactions on Consumer Electronics, 51(1), 230-234.

23. Yang, G., Wong, D., Wang, H., \& Deng, X. (2008). Two-factor mutual authentication based on smart cards and passwords. Journal of Computer and System Sciences, 74(7), 1160-1172.

24. Yang, G., Wong, D., \& Deng, X. (2007). Anonymous and authenticated key exchange for roaming networks. IEEE Transactions on Wireless Communications, 6(9), 1035-1042.

25. Karig, D. \& Lee, R. (2001). Remote Denial of Service Attacks and Countermeasures. Princeton University Department of Electrical Engineering Technical Report CE-L2001002. 